University of Montana

ScholarWorks at University of Montana

$5-2007$

\title{
Electronic Anisotropy Between Open Shell Atoms in First and Second Order Perturbation Theory
}

Gerrit C. Groenenboom

Xi Chu

University of Montana - Missoula, xi.chu@mso.umt.edu

R. Krems

Follow this and additional works at: https://scholarworks.umt.edu/chem_pubs

Part of the Biochemistry Commons, and the Chemistry Commons Let us know how access to this document benefits you.

\section{Recommended Citation}

Groenenboom, Gerrit C.; Chu, Xi; and Krems, R., "Electronic Anisotropy Between Open Shell Atoms in First and Second Order Perturbation Theory" (2007). Chemistry and Biochemistry Faculty Publications. 27. https://scholarworks.umt.edu/chem_pubs/27

This Article is brought to you for free and open access by the Chemistry and Biochemistry at ScholarWorks at University of Montana. It has been accepted for inclusion in Chemistry and Biochemistry Faculty Publications by an authorized administrator of ScholarWorks at University of Montana. For more information, please contact scholarworks@mso.umt.edu. 


\title{
Electronic anisotropy between open shell atoms in first and second order perturbation theory
}

\author{
Gerrit C. Groenenboom ${ }^{a)}$ \\ Theoretical Chemistry, Institute for Molecules and Materials, Radboud University Nijmegen, \\ Toernooiveld 1, 6525 ED Nijmegen, The Netherlands \\ $\mathrm{Xi} \mathrm{Chu}{ }^{\mathrm{b})}$ \\ Department of Chemistry, The University of Montana, Missoula, Montana 59812 \\ Roman V. Krems ${ }^{\mathrm{c})}$ \\ Department of Chemistry, University of British Columbia, Vancouver, British Columbia V6T 1Z1, Canada
}

(Received 9 November 2006; accepted 29 March 2007; published online 24 May 2007)

\begin{abstract}
The interaction between two atoms in states with nonzero electronic orbital angular momenta is anisotropic and can be represented by a spherical tensor expansion. The authors derive expressions for the first order (electrostatic) and second order (dispersion and induction) anisotropic interaction coefficients in terms of the multipole moments and dynamic polarizabilities of the atoms and show that a complete description of the second order interaction requires odd rank or "out-of-phase" polarizabilities. The authors relate the tensorial expansion coefficients to the adiabatic Born-Oppenheimer potentials of the molecule and show that there are linear, and in some cases nonlinear, constraints on the van der Waals coefficients of these potentials. (C) 2007 American Institute of Physics. [DOI: 10.1063/1.2733643]
\end{abstract}

\section{INTRODUCTION}

Recent experiments on magnetic trapping and cooling of atoms with nonzero electronic orbital angular momenta (non$S$-state atoms) to subkelvin temperatures ${ }^{1-4}$ have revived the interest in the long-range interaction between open shell atoms. Collisions at ultracold temperatures are determined by long-range interatomic forces and accurate calculations of van der Waals interaction coefficients between $S$-state alkali metal atoms proved to be invaluable for the experimental work with ultracold matter. ${ }^{5}$ The interaction of two atoms with nonzero electronic orbital angular momenta and nonzero electron spins gives rise to a manifold of adiabatic molecular potentials, the number and symmetries of which are given by the Wigner-Witmer rules. ${ }^{6}$ The molecular states with different total spins are well separated in energy due to exchange interactions at short range. At large interatomic separations, the exchange interactions vanish but the degeneracy of the molecular states may still be lifted by long-range electrostatic interactions. In addition, states with the same spin are coupled by radial and angular derivative nonadiabatic couplings. The accurate calculation of the adiabatic potentials and nonadiabatic couplings in this region is a difficult problem. The best methods to compute the long-range or van der Waals interactions between closed shell systems, such as supermolecular CCSD(T) with counterpoise basis set superposition error correction or symmetry adapted perturbation theory, are not generally applicable to open shell systems. Besides, these methods do not provide the couplings.

An alternative description of complexes involving open

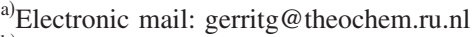

b) Electronic mail: xchu@chem.umt.edu

${ }^{c)}$ Electronic mail: rkrems@chem.ubc.ca
}

shell atoms, which avoids the computation of derivative couplings, is an approximate diabatic model. ${ }^{7-11}$ The number of diabatic states is chosen to be equal to the number of asymptotically degenerate adiabatic states. For example, the interaction between an oxygen atom in the ${ }^{3} P$ ground state with a helium atom is determined by adiabatic molecular potentials of $\Sigma$ and $\Pi$ symmetries. Callaway and Bauer ${ }^{7}$ proposed to model such an interaction by an effective potential acting in the subspace consisting of three $P$ substates. These substates are defined in a space-fixed frame with the origin on the open shell atom. Hence, the substates are not coupled by the nuclear kinetic energy operator which defines them as diabatic states. The effective potential that couples the substates may be called anisotropic, because it depends on the angle $\theta$ between the interatomic axis and the space-fixed $z$ axis. In the example of Callaway and Bauer, the effective potential can be expanded in Legendre polynomials and only zeroth and second order expansion terms are nonzero. The adiabatic potentials are obtained by diagonalization of the effective interaction potential matrix. In this case the transformation between the adiabatic potentials and the Legendre expansion coefficients is analytic. ${ }^{12,13}$ The description of the $\mathrm{O}$ atom with orbital angular momentum $L=1$ interacting with a helium atom is analogous to the interaction of a homonuclear diatomic molecule with rotational angular momentum $J=1$ interacting with helium.

Similar diabatic models have been used to describe the interaction of open shell atoms with $\Sigma$ state diatomic molecules. ${ }^{8,9,11}$ Based on these models, one might assume that the interaction between two non-S-state atoms with orbital angular momenta $L_{1}$ and $L_{2}$ can be represented in analogy to the interaction between two diatomic molecules with rotational angular momenta $J_{1}=L_{1}$ and $J_{2}=L_{2}$ as a spherical 
harmonic expansion. We have recently shown,${ }^{14}$ however, that this is not the case. We proposed a spherical tensor expansion for the interaction of two atoms in arbitrary states. The expansion contains both odd and even rank spherical tensors that are invariant under inversion. The even rank tensors can be represented by spherical harmonics of the same rank. However, the odd rank spherical tensors cannot be represented by odd rank spherical harmonics, since odd rank spherical harmonics have odd parity and their matrix elements in the model space are zero. Santra and Greene ${ }^{15}$ have recently presented a tensorial analysis of the long-range interaction between two $P$-state atoms. However, they employed a basis of coupled atomic states so the analogy with the diatomic-diatom interactions, and its breakdown, was not made transparent.

In the present paper, we use the spherical tensor expansion introduced in Ref. 14 to derive expressions for the first order electrostatic (Sec. II) and second order dispersion and induction (Sec. III) interactions between two open shell atoms and analyze the transformation to the adiabatic molecular states (Sec. IV). We show that the first order interaction has the same form as the anisotropic interaction between two closed shell diatomic molecules, whereas the second order interaction requires inversion invariant spherical tensors of odd rank. The interaction of an open shell atom with orbital angular momentum $L$ and a closed shell atom gives rise to $L+1$ distinct adiabatic potentials with $|\Lambda|=0,1, \ldots, L$, where $\Lambda$ is the electronic orbital angular momentum projection quantum number. The effective potential contains Legendre polynomials of order $k=0,2, \ldots, 2 L$ so the number of terms in the potential expansion is equal to the number of adiabatic potentials. We show that for two open shell atoms, this is no longer the case and the number of terms in the tensorial expansion of the effective potential may be larger than the number of adiabatic potentials. Furthermore, the transformation between the expansion coefficients and the adiabatic potentials is no longer fully determined by symmetry. We use the multipole expansion of the Coulomb interaction and derive expressions for the anisotropic van der Waals coefficients in terms of the permanent multipole moments and the static and frequency dependent polarizabilities of the atoms. We find that a complete description of the long-range interaction coefficients requires odd rank or "out-of-phase" 16 atomic polarizabilities in addition to the usual scalar and rank 2 tensor terms. In a separate paper the time-dependent density functional theory method is used to compute these atomic properties for $\operatorname{Sc}\left({ }^{1} D\right)$ and $\operatorname{Ti}\left({ }^{3} F\right)$. The expressions from the present paper are used to obtain the van der Waals coefficients for $\mathrm{Sc}-\mathrm{Sc}$, Ti-Ti, and $\mathrm{Sc}-\mathrm{Ti}^{17}$

The leading term in the long-range interaction of neutral non- $S$-state atoms $\left(\propto R^{-5}\right)$ is the quadrupole-quadrupole interaction. Apart from a single scaling parameter, the product of the atomic quadrupole moments, it is known analytically and the interatomic Born-Oppenheimer potentials are linearly related at large interatomic separations. These relations can be used to test interatomic potentials computed by, e.g., supermolecular methods and to find proper analytical forms to fit them. In this paper we show that the number of parameters for the interactions given by higher multipole moments and in second order may still be less than the number of molecular potentials. We determine the maximum number of independent parameters in the effective potentials (Sec. IV), and show how this number is reduced if we limit the $R^{-n}$ expansion of the interatomic potentials to terms with $n=5,6,7$, or 8 (Sec. V). Since the adiabatic potentials are obtained from diagonalization of the effective interaction Hamiltonian, the reduced number of parameters results in nonlinear constraints on the adiabatic potentials that can again be used to test or fit interatomic interaction potentials at long range. Section VI presents some examples and Sec. VII concludes.

\section{TENSOR FORMULATION OF ELECTROSTATIC INTERACTION}

The multipole expansion of the electrostatic Hamiltonian describing the interaction between atoms $A$ and $B$ can be written as ${ }^{18,19}$

$$
\begin{aligned}
\hat{H}_{\mathrm{el}}= & \sum_{l_{1} l_{2} M_{12}} \frac{(-1)^{l_{2}}}{R^{l_{1}+l_{2}+1}}\left(\begin{array}{c}
2 L_{12} \\
2 l_{1}
\end{array}\right)^{1 / 2} \\
& \times\left[\hat{Q}_{l_{1}}^{(A)} \otimes \hat{Q}_{l_{2}}^{(B)}\right]_{M_{12}}^{\left(L_{12}\right)} C_{L_{12} M_{12}}^{*}(\hat{\boldsymbol{R}}),
\end{aligned}
$$

where

$$
\left[\hat{Q}_{l_{1}}^{(A)} \otimes \hat{Q}_{l_{2}}^{(B)}\right]_{M_{12}}^{\left(L_{12}\right)}=\sum_{m_{1} m_{2}} \hat{Q}_{l_{1} m_{1}}^{(A)} \hat{Q}_{l_{2} m_{2}}^{(B)}\left\langle l_{1} m_{1} l_{2} m_{2} \mid L_{12} M_{12}\right\rangle
$$

is the irreducible rank- $L_{12}$ tensor product of $\hat{Q}_{l_{1} m_{1}}^{(A)}$ and $\hat{Q}_{l_{2} m_{2}}^{(B)}$, $C_{L_{12} M_{12}}$ is a Racah normalized spherical harmonic, $\left(\begin{array}{c}2 L_{12} \\ 2 l_{1}\end{array}\right)$ is a binomial coefficient, $L_{12}=l_{1}+l_{2}$, the symbol in angle brackets is a Clebsch-Gordan coefficient and $\hat{Q}_{l_{1} m_{1}}^{(A)}$ and $\hat{Q}_{l_{2} m_{2}}^{(B)}$ are the multipole moment operators of atoms $A$ and $B$ defined by

$$
\hat{Q}_{l_{1} m_{1}}^{(A)}=-e \sum_{i} r_{i}^{l_{1}} C_{l_{1} m_{1}}\left(\hat{r}_{i}\right)+e Z_{A} \delta_{l_{1}, 0}
$$

and an analogous expression for $\hat{Q}_{l_{2} m_{2}}^{(B)}$. The summation in Eq. (3) is over all electrons, $e$ is the elementary charge, and $Z_{A}$ is the atomic number of atom $A$. The vector $\boldsymbol{R}$ points from atom $A$ to atom $B$ in a space-fixed coordinate system, $R$ is the length of the vector, and $\hat{\boldsymbol{R}}=\boldsymbol{R} / R$. The electron coordinates $\boldsymbol{r}_{i}$ (with $r_{i}=\left|\boldsymbol{r}_{i}\right|$ and $\hat{\boldsymbol{r}}_{i}=\boldsymbol{r}_{i} / r_{i}$ ) are defined in frames with the coordinate axes parallel to the space-fixed frame and with origin on either atom $A$ or $B$.

We introduce a basis of atomic product states in the Russell-Saunders coupling scheme $\left|n_{A} L_{A} M_{A}\right\rangle\left|n_{B} L_{B} M_{B}\right\rangle$ where $L_{A}$ and $L_{B}$ are the electronic orbital quantum numbers for atoms $A$ and $B$ and $M_{A}$ and $M_{B}$ are the space-fixed projections of $L_{A}$ and $L_{B} ; n_{A}$ and $n_{B}$ represent all other quantum numbers including spin. We will omit them in the discussion below. The conventional procedure would be to compute matrix elements of the Hamiltonian $\hat{H}_{\mathrm{el}}$ in this basis and apply the Wigner-Eckart theorem to the matrix elements of the multipole moment operators 


$$
\begin{aligned}
\left\langle L_{A} M_{A}\left|\hat{Q}_{l_{1} m_{1}}^{(A)}\right| L_{A}^{\prime} M_{A}^{\prime}\right\rangle= & (-1)^{L_{A}-M_{A}}\left(\begin{array}{ccc}
L_{A} & l_{1} & L_{A}^{\prime} \\
-M_{A} & m_{1} & M_{A}^{\prime}
\end{array}\right) \\
& \times\left\langle L_{A}\left\|l_{1}\right\| L_{A}^{\prime}\right\rangle .
\end{aligned}
$$

All the Hamiltonian matrix elements would then be related to the product of reduced matrix elements $\left\langle L_{A}\left\|l_{1}\right\| L_{A}^{\prime}\right\rangle$ $\times\left\langle L_{B} \| l_{2} \mid L_{B}^{\prime}\right\rangle$.

In this paper, we use a more formal technique based on the expansion of the operators in terms of unit spherical tensor operators. This allows us to make the connection with the general spherical tensor description of the electronic interaction between two open-shell atoms introduced previously ${ }^{14}$ and makes the ensuing discussion of the second order interaction transparent. A good introduction to this formalism is given in the book of Blum. ${ }^{20}$ In this book spherical tensor operators are called irreducible tensor operators.

We define a primitive operator basis for atom $A$

$$
\hat{\tau}_{M_{A} M_{A}^{\prime}}\left(L_{A} ; L_{A}^{\prime}\right)=\left|L_{A} M_{A}\right\rangle\left\langle L_{A}^{\prime} M_{A}^{\prime}\right|
$$

and expand the multipole moment operator in this basis

$$
\hat{Q}_{l_{1} m_{1}}^{(A)}=\sum_{L_{A} M_{A} ; L_{A}^{\prime} M_{A}^{\prime}} \hat{\tau}_{M_{A} M_{A}^{\prime}}\left(L_{A} ; L_{A}^{\prime}\right) Q_{l_{1} m_{1}}^{(A)}\left(L_{A} M_{A} ; L_{A}^{\prime} M_{A}^{\prime}\right) .
$$

The expansion coefficients are equal to the matrix elements

$$
Q_{l_{1} m_{1}}^{(A)}\left(L_{A} M_{A} ; L_{A}^{\prime} M_{A}^{\prime}\right)=\left\langle L_{A} M_{A}\left|\hat{Q}_{l_{1} m_{1}}^{(A)}\right| L_{A}^{\prime} M_{A}^{\prime}\right\rangle .
$$

The operators $\hat{\tau}_{M_{A} M_{A}^{\prime}}\left(L_{A} ; L_{A}^{\prime}\right)$ are elements of a linear space. A scalar product for any two elements $\hat{\tau}_{1}$ and $\hat{\tau}_{2}$ of this space is defined as ${ }^{20}$

$$
\left\langle\hat{\tau}_{1} \mid \hat{\tau}_{2}\right\rangle=\operatorname{trace}\left\{\hat{\tau}_{1}^{\dagger} \hat{\tau}_{2}\right\}=\sum_{L_{A} M_{A}}\left\langle L_{A} M_{A}\left|\hat{\tau}_{1}^{\dagger} \hat{\tau}_{2}\right| L_{A} M_{A}\right\rangle .
$$

The basis $\hat{\tau}_{M_{A} M_{A}^{\prime}}\left(L_{A} ; L_{A}^{\prime}\right)$ is called "unit" or orthonormal because

$$
\begin{gathered}
\left\langle\hat{\tau}_{M_{A} M_{A}^{\prime}}\left(L_{A} ; L_{A}^{\prime}\right) \mid \hat{\tau}_{M_{A}^{\prime \prime} M_{A}^{\prime \prime \prime}}\left(L_{A}^{\prime \prime} ; L_{A}^{\prime \prime \prime}\right)\right\rangle \\
=\delta_{L_{A} L_{A}^{\prime \prime}} \delta_{M_{A} M_{A}^{\prime \prime}} \delta_{L_{A}^{\prime} L_{A}^{\prime \prime \prime}} \delta_{M_{A}^{\prime} M_{A}^{\prime \prime \prime} \cdot}
\end{gathered}
$$

A unit spherical tensor operator basis is defined by a unitary transformation of the primitive basis

$$
\hat{T}_{l_{1} m_{1}}\left(L_{A} ; L_{A}^{\prime}\right)=\sum_{M_{A} M_{A}^{\prime}} \hat{\tau}_{M_{A} M_{A}^{\prime}}\left(L_{A} ; L_{A}^{\prime}\right) \mathcal{U}_{M_{A} M_{A}^{\prime} ; l_{1} m_{1}}^{\left(L_{L_{1}}^{\prime}\right)}
$$

where the matrix elements of the unitary transformation are given by

$$
\begin{aligned}
& \mathcal{U}_{M_{A} M_{A}^{\prime} ; l_{1} m_{1}}^{\left(L_{A} ; L_{A}^{\prime}\right)}=(-1)^{L_{A}-M_{A}}\left(\begin{array}{ccc}
L_{A} & l_{1} & L_{A}^{\prime} \\
-M_{A} & m_{1} & M_{A}^{\prime}
\end{array}\right)\left[l_{1}\right]^{1 / 2} \\
& =(-1)^{2 l_{1}+L_{A}^{\prime}-M_{A}^{\prime}}\left\langle L_{A}, M_{A}, L_{A}^{\prime},-M_{A}^{\prime} \mid l_{1}, m_{1}\right\rangle,
\end{aligned}
$$

with $\left[l_{1}\right] \equiv 2 l_{1}+1$.

Since the multipole moment operator is a spherical tensor operator it can be expanded as

$$
\hat{Q}_{l_{1} m_{1}}^{(A)}=\sum_{L_{A} ; L_{A}^{\prime}} \hat{T}_{l_{1} m_{1}}\left(L_{A} ; L_{A}^{\prime}\right) Q_{l_{1}}^{(A)}\left(L_{A} ; L_{A}^{\prime}\right) .
$$

By substituting Eq. (10) into this equation and comparing the result with Eq. (6) one finds the unitary transformation between the expansion coefficients,

$$
Q_{l_{1} m_{1}}^{(A)}\left(L_{A} M_{A} ; L_{A}^{\prime} M_{A}^{\prime}\right)=Q_{l_{1}}^{(A)}\left(L_{A} ; L_{A}^{\prime}\right) \mathcal{U}_{M_{A} M_{A}^{\prime} ; l_{1} m_{1}}^{\left(L_{L_{1}} ; L^{\prime}\right)} .
$$

The expansion coefficient in the spherical basis is proportional to the reduced matrix element from the Wigner-Eckart theorem

$$
Q_{l_{1}}^{(A)}\left(L_{A} ; L_{A}^{\prime}\right)=\left[l_{1}\right]^{-1 / 2}\left\langle L_{A}\left\|l_{1}\right\| L_{A}^{\prime}\right\rangle .
$$

The first and second order perturbation operators act in a space with fixed quantum numbers $L_{A}$ and $L_{B}$ so we introduce the notation

$\hat{T}_{l_{1} m_{1}}\left(L_{A}\right) \equiv \hat{T}_{l_{1} m_{1}}\left(L_{A} ; L_{A}\right) \quad$ and $\quad Q_{l_{1}}^{(A)}\left(L_{A}\right) \equiv Q_{l_{1}}^{(A)}\left(L_{A} ; L_{A}\right)$.

The Hamiltonian for the first order electrostatic interaction can now be written in a coupled spherical tensor form as

$$
\hat{H}_{e l}^{(1)}=\sum_{l_{1} l_{2}} \frac{c_{n,\left(l_{1} l_{2}\right) L_{12}}^{(1)} \sum^{L_{12}+1}}{M_{12}}\left[\hat{T}_{l_{1}}\left(L_{A}\right) \otimes \hat{T}_{l_{2}}\left(L_{B}\right)\right]_{M_{12}}^{\left(L_{12}\right)} C_{L_{12} M_{12}}^{*}(\hat{\boldsymbol{R}}),
$$

where $n=L_{12}+1$ and the coefficients of the first order electrostatic interaction are fully determined by the atomic multipole moments,

$$
c_{n,\left(l_{1} l_{2}\right) L_{12}}^{(1)}=(-1)^{l_{2}}\left(\begin{array}{c}
2 L_{12} \\
2 l_{2}
\end{array}\right)^{1 / 2} Q_{l_{1}}^{(A)}\left(L_{A}\right) Q_{l_{2}}^{(B)}\left(L_{B}\right) .
$$

\section{DISPERSION AND INDUCTION INTERACTIONS}

The second order perturbation operator that gives rise to the long-range induction and dispersion interactions can be written in terms of the electronic Hamiltonian as follows:

$$
\hat{H}^{(2)}=\hat{H}_{\mathrm{el}} \hat{G} \hat{H}_{\mathrm{el}},
$$

with the reduced resolvent $\hat{G}$ defined as

$$
\hat{G}=-\sum_{a b}, \frac{|a b\rangle\langle a b|}{\Delta E_{a}+\Delta E_{b}},
$$

where $a$ and $b$ denote all quantum numbers of atoms $A$ and $B$, respectively, and $\Delta E_{a}=E_{a}-E_{a_{0}}$ and $\Delta E_{b}=E_{b}-E_{b_{0}}$ are the excitation energies of atoms $A$ and $B$. The prime on the summation sign indicates that terms with both $\Delta E_{a}=0$ and $\Delta E_{b}$ $=0$ are excluded. We do not consider resonant interactions for which $\Delta E_{a}=-\Delta E_{b}$.

The substitution of Eqs. (1) and (19) into (18) gives, after some angular momentum algebra, 


$$
\begin{aligned}
\hat{H}^{(2)}= & \sum_{l_{1} l_{2} l_{1}^{\prime} l_{2}^{\prime} k_{1} k_{2} k q} \frac{-f_{l_{1} l_{1}^{\prime} l_{2} l_{2}^{\prime}}^{\left(k_{2} k_{k} k\right)} C_{k q}^{*}(\hat{\boldsymbol{R}})}{R^{L_{12}+L_{12}^{\prime}+2}} \\
& \times \sum_{a b}, \frac{\left[\left[\hat{Q}_{l_{1}}^{(A)}|a\rangle\langle a| \hat{Q}_{l_{1}^{\prime}}^{(A)}\right]^{\left(k_{1}\right)} \otimes\left[\hat{Q}_{l_{2}}^{(B)}|b\rangle\langle b| \hat{Q}_{l_{2}^{\prime}}^{(B)}\right]^{\left(k_{2}\right)}\right]_{q}^{(k)}}{\Delta E_{a}+\Delta E_{b}},
\end{aligned}
$$

where $L_{12}=l_{1}+l_{2}, L_{12}^{\prime}=l_{1}^{\prime}+l_{2}^{\prime}$, and

$$
\begin{aligned}
f_{l_{1} l_{1}^{\prime} l_{2}^{\prime} l_{2}}^{\left(k_{1} k_{2} k\right)} & (-1)^{l_{2}+l_{2}^{\prime}}\left(\begin{array}{c}
2 L_{12} \\
2 l_{1}
\end{array}\right)^{1 / 2}\left(\begin{array}{c}
2 L_{12}^{\prime} \\
2 l_{1}^{\prime}
\end{array}\right)^{1 / 2}\left\langle L_{12} 0 L_{12}^{\prime} 0 \mid k 0\right\rangle \\
& \times\left[L_{12}, L_{12}^{\prime}, k_{1}, k_{2}\right]^{1 / 2}\left\{\begin{array}{ccc}
l_{1} & l_{1}^{\prime} & k_{1} \\
l_{2} & l_{2}^{\prime} & k_{2} \\
L_{12} & L_{12}^{\prime} & k
\end{array}\right\},
\end{aligned}
$$

the symbol in curly brackets is a $9-j$ symbol. The terms with either $\Delta E_{a}=0$ or $\Delta E_{b}=0$ give the induction interactions.

We first consider the dispersion interaction. Following Casimir and Polder, ${ }^{21}$ we use the relation

$$
\frac{1}{A+B}=\frac{1}{2 \pi} \int_{0}^{\infty} \frac{2 A}{A^{2}+\omega^{2}} \frac{2 B}{B^{2}+\omega^{2}} d \omega
$$

to rewrite the second order Hamiltonian as

$$
\begin{aligned}
\hat{H}^{(2)}= & \sum_{l_{1} l_{2} l_{1}^{\prime} l_{2}^{\prime} k_{1} k_{2} k q} \frac{-f_{l_{1} l_{1}^{\prime} l_{2} l_{2}^{\prime}}^{\left(k_{k} k_{k q}\right)} C_{k q}^{*}(\hat{\boldsymbol{R}})}{R^{L_{12}+L_{12}^{\prime}+2}} \\
& \times \sum_{q_{1} q_{2}} \frac{1}{2 \pi} \int_{0}^{\infty} \hat{\alpha}_{\left(l_{1} l_{1}^{\prime}\right) k_{1} q_{1}}^{(A)}(i \omega) \hat{\alpha}_{\left(l_{2} l_{2}^{\prime}\right) k_{2} q_{2}}^{(B)}(i \omega) d \omega \\
& \times\left\langle k_{1} q_{1} k_{2} q_{2} \mid k q\right\rangle,
\end{aligned}
$$

where the spherical dynamic polarizability operators are defined by

$$
\hat{\alpha}_{\left(l_{1} l_{1}^{\prime}\right) k_{1} q_{1}}=\sum_{m_{1} m_{1}^{\prime}} \hat{\alpha}_{l_{1} m_{1} ; l_{1}^{\prime} m_{1}^{\prime}}\left\langle l_{1} m_{1} l_{1}^{\prime} m_{1}^{\prime} \mid k_{1} q_{1}\right\rangle
$$

and

$$
\hat{\alpha}_{l_{1} m_{1} ; l_{1}^{\prime} m_{1}^{\prime}}^{(A)}(i \omega) \equiv \sum_{a}^{\prime} \frac{2 \Delta E_{a} \hat{Q}_{l_{1} m_{1}}^{(A)}|a\rangle\langle a| \hat{Q}_{l_{1}^{\prime} m_{1}^{\prime}}^{(A)}}{\Delta E_{a}^{2}+\omega^{2}} .
$$

Only even rank polarizability operators contribute to Eq. (23) when one of the atoms is in an $S$-state, i.e., when $L_{B}$ $=0$ then $k_{2}=0$ and $k_{1}=k$ must be even (see Sec. VI). For two non- $S$-state atoms, there is a nonzero contribution from odd rank polarizabilities. To elucidate the role of such odd rank polarizabilities, we introduce the symmetrized operators $\hat{\alpha}_{l_{1} m_{1} ; l_{1}^{\prime} m_{1}^{\prime}}^{ \pm}$as follows:

$$
\hat{\alpha}_{l_{1} m_{1} ; l_{1}^{\prime} m_{1}^{\prime}}^{ \pm} \equiv \frac{\hat{\alpha}_{l_{1} m_{1} ; l_{1}^{\prime} m_{1}^{\prime}} \pm \hat{\alpha}_{l_{1}^{\prime} m_{1}^{\prime} ; l_{1} m_{1}}}{2} .
$$

The expectation values of the operator $\hat{\alpha}^{+}$in the ground electronic states are the usual dynamic polarizabilities. We will refer to the operator $\hat{\alpha}^{-}$as the "out-of-phase" polarizability.
The spherical components of $\hat{\alpha}^{ \pm}$are given by

$$
\hat{\alpha}_{\left(l_{1} l_{1}^{\prime}\right) k_{1} q_{1}}^{ \pm} \equiv \sum_{m_{1} m_{1}^{\prime}} \hat{\alpha}_{l_{1} m_{1} ; l_{1}^{\prime} m_{1}^{\prime}}^{ \pm}\left\langle l_{1} m_{1} l_{1}^{\prime} m_{1}^{\prime} \mid k_{1} q_{1}\right\rangle
$$

Using Eqs. (24), (26), and (27) we express the unsymmetrized spherical polarizabilities as

$$
\hat{\alpha}_{\left(l_{1} l_{1}^{\prime}\right) k_{1} q_{1}}=\hat{\alpha}_{\left(l_{1} l_{1}^{\prime}\right) k_{1} q_{1}}^{+}+\hat{\alpha}_{\left(l_{1} l_{1}^{\prime}\right) k_{1} q_{1}}^{-} .
$$

The operators introduced have the following properties:

$$
\begin{aligned}
& \hat{\alpha}_{l_{1} m_{1} ; l_{1}^{\prime} m_{1}^{\prime}}^{ \pm}= \pm \hat{\alpha}_{l_{1}^{\prime} m_{1}^{\prime} ; l_{1} m_{1}}^{ \pm}, \\
& \hat{\alpha}_{\left(l_{1} l_{1}^{\prime}\right) k_{1} q_{1}}^{ \pm}= \pm(-1)^{l_{1}+l_{1}^{\prime}+k_{1}} \hat{\alpha}_{\left(l_{1}^{\prime} l_{1}\right) k_{1} q_{1}}^{ \pm}, \\
& \hat{\alpha}_{l_{1} m_{1} ; l_{1}^{\prime} m_{1}^{\prime}}^{\dagger}=(-1)^{m_{1}+m_{1}^{\prime}} \hat{\alpha}_{l_{1}^{\prime},-m_{1}^{\prime} ; l_{1},-m_{1}}, \\
& {\left[\hat{\alpha}_{\left(l_{1} l_{1}^{\prime}\right) k_{1} q_{1}}^{ \pm}\right]^{\dagger}= \pm(-1)^{l_{1}+l_{1}^{\prime}+k_{1}-q_{1}} \hat{\alpha}_{\left(l_{1} l_{1}^{\prime}\right) k_{1},-q_{1}}^{ \pm} .}
\end{aligned}
$$

Equation (30) shows that for $l_{1}=l_{1}^{\prime}$ the operator $\hat{\alpha}_{\left(l_{1} l_{1}\right) k_{1} q_{1}}^{+}$vanishes unless $k_{1}$ is even and $\hat{\alpha}_{\left(l_{1} l_{1}\right) k_{1} q_{1}}$ vanishes unless $k_{1}$ is odd. Furthermore, since the parity of the multipole moment operator $\hat{Q}_{l m}(L)$ is $(-1)^{l}$, the parity of the polarizability operators is $l_{1}+l_{1}^{\prime}$. Hence, for any given atomic state $l_{1}+l_{1}^{\prime}$ must be even. Equation (32) shows that for $q_{1}=0$, the operators $\hat{\alpha}_{\left(l_{1} l_{1}\right) k_{1} 0}^{ \pm}$are Hermitian.

The operator $\hat{\alpha}_{\left(l_{1} l_{1}^{\prime}\right) k_{1} q_{1}}^{ \pm}$is a spherical tensor operator so it can be expanded similarly to the multipole moment operator in the previous section,

$$
\begin{aligned}
\hat{\alpha}_{\left(l_{1} l_{1}^{\prime}\right) k_{1} q_{1}}^{ \pm} & =\hat{T}_{k_{1} q_{1}}\left(L_{A}\right) \alpha_{\left(l_{1} l_{1}^{\prime}\right) k_{1}}^{ \pm}\left(L_{A}\right) \\
& =\sum_{M_{A} M_{A}^{\prime}} \hat{\tau}_{M_{A} M_{A}^{\prime}}\left(L_{A}\right) \alpha_{\left(l_{1} l_{1}^{\prime}\right) k_{1} q_{1}}^{ \pm}\left(L_{A} M_{A} ; L_{A} M_{A}^{\prime}\right) .
\end{aligned}
$$

The expansion coefficients in the primitive basis are the usual polarizabilities and the expansion coefficients in the unit spherical tensor basis are related to the reduced matrix elements in the Wigner-Eckart theorem as before,

$$
\alpha_{\left(l_{1} l_{1}^{\prime}\right) k_{1} q_{1}}^{ \pm}\left(L_{A} M_{A} ; L_{A} M_{A}^{\prime}\right)=\alpha_{\left(l_{1} l_{1}^{\prime}\right) k_{1}}^{ \pm}\left(L_{A}\right) \mathcal{U}_{M_{A} M_{A}^{\prime} ; k_{1} q_{1}}^{\left(L_{A}: L_{A}\right)} .
$$

The Hermitian conjugate of a spherical tensor operator is given by

$$
\hat{T}_{k_{1} q_{1}}^{\dagger}\left(L_{A}\right)=(-1)^{q_{1}} \hat{T}_{k_{1},-q_{1}}\left(L_{A}\right) .
$$

If we substitute this and Eq. (33) into (32) we find that

$$
\alpha_{\left(l_{1} l_{1}^{\prime}\right) k_{1}}^{ \pm}\left(L_{A}\right)^{*}= \pm(-1)^{k} \alpha_{\left(l_{1} l_{1}^{\prime}\right) k_{1}}^{ \pm},
$$

and hence for $l_{1}=l_{1}^{\prime}$ we find that $\alpha_{\left(l_{1} l_{1}\right) k_{1}}^{ \pm}\left(L_{A}\right)$ is real.

The second order interaction Hamiltonian [Eq. (20)] can be written in a coupled spherical tensor form as 


$$
\hat{H}^{(2)}=\sum_{n k_{1} k_{2} k} \frac{-c_{n,\left(k_{1} k_{2}\right) k}^{(2)}}{R^{n}} \sum_{q}\left[\hat{T}_{k_{1}}\left(L_{A}\right) \otimes \hat{T}_{k_{2}}\left(L_{B}\right)\right]_{q}^{(k)} C_{k q}^{*}(\hat{\boldsymbol{R}}),
$$

where

$$
c_{n,\left(k_{1} k_{2}\right) k}^{(2)}=c_{n,\left(k_{1} k_{2}\right) k}^{(\mathrm{disp})}+c_{n,\left(k_{1} k_{2}\right) k}^{(\mathrm{ind}, \mathrm{A})}+c_{n,\left(k_{1} k_{2}\right) k}^{(\mathrm{ind}, \mathrm{B})} .
$$

The dispersion contribution to the second order interaction coefficients is given by

$$
\begin{aligned}
c_{n,\left(k_{1} k_{2}\right) k}^{(\text {disp })}= & \sum_{l_{1} l_{2} l_{1}^{\prime} l_{2}^{\prime}} \frac{f_{l_{1} l_{1}^{\prime} l_{2} l_{2}^{\prime}}^{\left(k_{1} k_{2} k\right)} \int_{0}^{\infty} \alpha_{\left(l_{1} l_{1}^{\prime}\right) k_{1}}^{(A)}\left(L_{A} ; i \omega\right)}{2 \pi} \\
& \times \alpha_{\left(l_{2} l_{2}^{\prime}\right) k_{2}}^{(B)}\left(L_{B} ; i \omega\right) d \omega,
\end{aligned}
$$

with the sum restricted to terms with $l_{1}+l_{2}+l_{1}^{\prime}+l_{2}^{\prime}+2=n$. The polarizabilities $\alpha^{(A)}$ and $\alpha^{(B)}$ are obtained from the symmetrized polarizabilities [Eq. (26)],

$$
\alpha_{\left(l_{1} l_{1}^{\prime}\right) k_{1}}\left(L_{A}\right)=\alpha_{\left(l_{1} l_{1}^{\prime}\right) k_{1}}^{+}\left(L_{A}\right)+\alpha_{\left(l_{1} l_{1}^{\prime}\right) k_{1}}^{-}\left(L_{A}\right) .
$$

The following three relations will be useful for the computation of the polarizabilities:

$$
\begin{aligned}
& \sum_{m_{1} m_{1}^{\prime}} \alpha_{l_{1} m_{1} ; l_{1}^{\prime} m_{1}^{\prime}}^{ \pm}\left(L_{A} M_{A} ; L_{A} M_{A}^{\prime}\right)\left\langle l_{1} m_{1} l_{1}^{\prime} m_{1}^{\prime} \mid k_{1} q_{1}\right\rangle \\
& \quad=\mathcal{U}_{M_{A} M_{A}^{\prime} ; k_{1} q_{1}}^{\left(L_{1} ; L_{A}\right)} \alpha_{\left(l_{1} l_{1}^{\prime}\right) k_{1}}^{ \pm}\left(L_{A}\right) .
\end{aligned}
$$

Using the unitarity of Clebsch-Gordan coefficients we invert this equation to find

$$
\begin{aligned}
& \alpha_{l_{1} m_{1} ; l_{1}^{\prime} m_{1}^{\prime}}^{ \pm}\left(L_{A} M_{A} ; L_{A} M_{A}^{\prime}\right) \\
& \quad=\sum_{k_{1} q_{1}}\left\langle l_{1} m_{1} l_{1}^{\prime} m_{1}^{\prime} \mid k_{1} q_{1}\right\rangle \mathcal{M}_{M_{A} M_{A}^{\prime} ; k_{1} q_{1}}^{\left(L_{A} ; L_{A}\right)} \alpha_{\left(l_{1} l_{1}^{\prime}\right) k_{1}}^{ \pm}\left(L_{A}\right)
\end{aligned}
$$

and

$$
\begin{aligned}
& \sum_{M_{A} M_{A}^{\prime}} \alpha_{l_{1} m_{1} ; l_{1}^{\prime} m_{1}^{\prime}}^{ \pm}\left(L_{A} M_{A} ; L_{A} M_{A}^{\prime}\right) \mathcal{U}_{M_{A} M_{A}^{\prime} ; k_{1} q_{1}}^{\left(L_{A}: L_{A}\right)} \\
& \quad=\left\langle l_{1} m_{1} l_{1}^{\prime} m_{1}^{\prime} \mid k_{1} q_{1}\right\rangle \alpha_{\left(l_{1} l_{1}^{\prime}\right) k_{1}}^{ \pm}\left(L_{A}\right) .
\end{aligned}
$$

The induction contribution is obtained from Eq. (20) by restricting the sum over $b$ to $|b\rangle=\left|L_{B} M_{B}\right\rangle$ and setting $\Delta E_{b}$ $=0$. We define

$$
\hat{Q}_{\left(l_{2} l_{2}^{\prime}\right) k_{2} q_{2}}^{(B)}\left(L_{B}\right) \equiv\left[\hat{Q}_{l_{2}}\left(L_{B}\right) \hat{Q}_{l_{2}^{\prime}}\left(L_{B}\right)\right]_{q_{2}}^{\left(k_{2}\right)},
$$

where we used the notation of Eq. (2) without the " $\otimes$ " symbol because the operators are acting on the same space. With the relation

$$
\hat{Q}_{\left(l_{2} l_{2}^{\prime}\right) k_{2} q_{2}}^{(B)}=\hat{T}_{k_{2} q_{2}}\left(L_{\mathrm{B}}\right) Q_{\left(l_{2} l_{2}^{\prime}\right) k_{2}}^{(B)},
$$

we find for the induction coefficient

$$
c_{n,\left(k_{1} k_{2}\right) k}^{(\text {ind,A) }}=\frac{1}{2} \sum_{l_{1} l_{2} l_{1}^{\prime} l_{2}^{\prime}} f_{l_{1} l_{1}^{\prime} l_{2} l_{2}^{\prime}}^{\left(k_{2} k_{2} k\right)} \alpha_{\left(l_{1} l_{1}^{\prime}\right) k_{1}}^{(A)}\left(L_{A} ; \omega=0\right) Q_{\left(l_{2} l_{2}^{\prime}\right) k_{2}}^{(B)}\left(L_{\mathrm{B}}\right)
$$

and an analogous expression for $c_{n,\left(k_{1} k_{2}\right) k}^{(\text {ind, })}$. Substituting $\hat{Q}_{l_{2} m_{2}}\left(L_{B}\right)=\hat{T}_{l_{2} m_{2}}\left(L_{B}\right) Q_{l_{2}}\left(L_{B}\right)$ into Eq. (44) and using the general tensor relation Eq. A4 from Ref. 12 we can relate the coupled multipole moment $Q_{\left(l_{2} l_{2}^{\prime}\right) k_{2}}^{(B)}\left(L_{\mathrm{B}}\right)$ to the multipole moment $Q_{l_{2}}\left(L_{B}\right)$ as

$$
\begin{aligned}
Q_{\left(l_{2} l_{2}^{\prime}\right) k_{2}}^{(B)}\left(L_{\mathrm{B}}\right)= & Q_{l_{2}}\left(L_{B}\right) Q_{l_{2}^{\prime}}\left(L_{B}\right) \\
& \times(-1)^{k_{2}}\left[l_{2}, l_{2}^{\prime}\right]^{1 / 2}\left\{\begin{array}{ccc}
L_{B} & L_{B} & l_{2} \\
l_{2}^{\prime} & k_{2} & L_{B}
\end{array}\right\},
\end{aligned}
$$

where the symbol in the curly brackets is a $6-j$ symbol.

\section{RELATION WITH THE MOLECULAR POTENTIALS}

In a recent paper ${ }^{14}$ we presented the general form of the long-range interaction between two atoms in RussellSaunders coupled states with electronic orbital angular momenta $L_{A}$ and $L_{B}$. When the spin-orbit interaction is ignored, the effective interaction potential can be written as

$$
\hat{V}=\sum_{S \Sigma}|S \Sigma\rangle\langle S \Sigma| \hat{V}^{S}
$$

where

$$
\hat{V}^{S}=\sum_{k_{1} k_{2} k} V_{k_{1} k_{2} k}^{S}(R) \sum_{q}\left[\hat{T}_{k_{1}}\left(L_{\mathrm{A}}\right) \otimes \hat{T}_{k_{2}}\left(L_{\mathrm{B}}\right)\right]_{q}^{(k)} C_{k q}^{*}(\hat{\boldsymbol{R}}),
$$

and $S$ and $\Sigma$ are the total electron spin and its projection on the interatomic axis. The expansion coefficients $V_{k_{1} k_{2} k}^{S}$ in the space-fixed basis of coupled unit spherical tensor operators are related to the matrix elements $V_{L \Lambda ; L^{\prime} \Lambda}^{S}(R)$ of the effective interaction potential $\hat{V}^{S}$ in a body-fixed coupled atomic basis

$$
\left|\left(L_{A} L_{B}\right) L \Lambda\right\rangle=\sum_{\Lambda_{A} \Lambda_{B}}\left|L_{A} \Lambda_{A} L_{B} \Lambda_{B}\right\rangle\left\langle L_{A} \Lambda_{A} L_{B} \Lambda_{B} \mid L \Lambda\right\rangle
$$

by the expression

$$
\begin{aligned}
V_{k_{1} k_{2} k}^{S}(R)= & \sum_{L L^{\prime} \Lambda} V_{L \Lambda ; L^{\prime} \Lambda}^{S}(R)(-1)^{L-\Lambda}\left(\begin{array}{ccc}
L & k & L^{\prime} \\
-\Lambda & 0 & \Lambda
\end{array}\right) \\
& \times\left[k_{1}, k_{2}, k, L, L^{\prime}\right]^{1 / 2}\left\{\begin{array}{ccc}
L_{\mathrm{A}} & L_{\mathrm{A}} & k_{1} \\
L_{\mathrm{B}} & L_{\mathrm{B}} & k_{2} \\
L & L^{\prime} & k
\end{array}\right\} .
\end{aligned}
$$

The projection of the total electronic orbital angular momentum $\Lambda=\Lambda_{A}+\Lambda_{B}$ is conserved. The adiabatic BornOppenheimer (ABO) potentials for a given $S$ and $\Lambda$ are obtained by diagonalization of the effective interaction matrix with elements $V_{L \Lambda: L^{\prime} \Lambda}^{S}(R)$, for $L, L^{\prime}=\left|L_{A}-L_{B}\right|, \ldots, L_{A}+L_{B}$. Since the eigenvectors of this matrix may depend on $R$ the effective interaction operator also describes the radial derivative couplings between ABO states with the same $S$ and $\Lambda$. 
TABLE I. The symmetries of (singlet) molecular states $\left\{\left|\left(L_{A} L_{B}\right) L \Lambda\right\rangle, L\right.$ $\left.=\left|L_{A}-L_{B}\right|, \ldots, L_{A}+L_{B}\right\}$ [Eq. (50)] for two even parity atoms and zero total electron spin. The $g / u$ labels apply only to homonuclear molecules.

\begin{tabular}{lccccc}
\hline \hline$L \backslash \Lambda$ & 0 & 1 & 2 & 3 & 4 \\
\hline 0 & ${ }^{1} \Sigma_{g}^{+}$ & & & & \\
1 & ${ }^{1} \Sigma_{u}^{-}$ & ${ }^{1} \Pi_{u}$ & & & \\
2 & ${ }^{1} \Sigma_{g}^{+}$ & ${ }^{1} \Pi_{g}$ & ${ }^{1} \Delta_{g}$ & & \\
3 & ${ }^{1} \Sigma_{u}^{-}$ & ${ }^{1} \Pi_{u}$ & ${ }^{1} \Delta_{u}$ & ${ }^{1} \Phi_{u}$ & \\
4 & ${ }^{1} \Sigma_{g}^{+}$ & ${ }^{1} \Pi_{g}$ & ${ }^{1} \Delta_{g}$ & ${ }^{1} \Phi_{g}$ & ${ }^{1} \Gamma_{g}$ \\
\hline \hline
\end{tabular}

\section{A. Independent parameters}

Equation (51) constitutes a unitary transformation so the number of independent body-fixed potential parameters $V_{L \Lambda ; L^{\prime} \Lambda}^{S}(R)$ is equal to the number of independent space-fixed parameters $V_{k_{1} k_{2} k}^{S}(R)$. Here, we determine the maximum number of independent parameters for an effective operator acting in the space spanned by

$$
\left|L_{A} \Lambda_{A} L_{B} \Lambda_{B}\right\rangle, \Lambda_{A}=-L_{A}, \ldots, L_{A} ; \Lambda_{B}=-L_{B}, \ldots, L_{B},
$$

taking into account the Hermiticity of the interaction operator and its invariance under time reversal and space inversion. Due to the cylindrical symmetry $\left(C_{\infty}\right)$, the projection of the electronic orbital angular momentum on the body-fixed axis $\Lambda=\Lambda_{A}+\Lambda_{B}$ is a good quantum number. For $L_{A}=L_{B}$ we will distinguish homonuclear and heteronuclear molecules, i.e., $D_{\infty, h}$ and $C_{\infty, v}$ point group symmetries. We do not explicitly take into account the anti-symmetry of the wave functions with respect to electron interchange, because we consider interactions at long range. Note, however, that for homonuclear molecules $L+S$ must be even (odd) for gerade (ungerade) states. ${ }^{22}$

The dimension of the space spanned by the uncoupled basis in Eq. (52) or the coupled basis Eq. (50) is $m=\left(2 L_{A}\right.$ $+1)\left(2 L_{B}+1\right)$. An effective Hamiltonian acting in this space has $m^{2}$ matrix elements. If the Hamiltonian is invariant under time reversal, a phase convention can be chosen to make the Hamiltonian matrix real. ${ }^{23}$ In addition, if the Hamiltonian is Hermitian the maximum number of independent parameters is $m(m+1) / 2$. This number is further reduced if we take into account $C_{\infty, v}$ or $D_{\infty, h}$ symmetry, because matrix elements between basis functions that belong to different irreducible representations (irreps) of these groups are zero. The counting is most easily performed in the coupled basis. In the phase convention of Condon and Shortley the matrix elements $V_{L \Lambda ; L^{\prime} \Lambda}^{S}$ are real and hence the Hermiticity of the interaction operator gives

$$
V_{L \Lambda ; L^{\prime} \Lambda}^{S}=\left(V_{L^{\prime} \Lambda ; L \Lambda}^{S}\right)^{*}=V_{L^{\prime} \Lambda ; L \Lambda}^{S} .
$$

As an intermediate step we first consider $C_{\infty}$ symmetry.

\section{B. $C_{\infty}$ symmetry}

In this group the irreps are labeled by $\Lambda$. Below, we use $L_{<}=\min \left(L_{A}, L_{B}\right), \quad L_{>}=\max \left(L_{A}, L_{B}\right), \quad$ and $N=L_{>}-L_{<}=\mid L_{A}$ $-L_{B} \mid$. The number of states for a given value of $\Lambda$ when $|\Lambda| \leqslant N$ is $2 L_{<+1}$. These states require $(2 N+1)\left(2 L_{<}+1\right)$ $\times\left(L_{<}+1\right)$ parameters (see Table I). The remaining states with $|\Lambda|>N$ require

$$
2 \sum_{i=1}^{2 L_{<}} \frac{i}{2}(i+1)=\frac{4}{3} L_{<}\left(L_{<}+1\right)\left(2 L_{<}+1\right)
$$

parameters, where we used the sum formulas given in Appendix A. The total number of parameters in $C_{\infty}$ symmetry is

$$
n\left(C_{\infty}\right)=\left(L_{<}+1\right)\left(2 L_{<}+1\right)\left[\frac{4}{3} L_{<}+2 N+1\right] .
$$

\section{C. $\boldsymbol{C}_{\infty, v}$ symmetry}

Space-fixed inversion of electronic and nuclear coordinates results in the reflection $\left(\hat{\sigma}_{v}\right)$ of the electronic coordinates in the body-fixed frame (see, e.g., the appendix of Ref. 24). We assume that

$$
\hat{\sigma}_{v}\left|L_{A} \Lambda_{A}\right\rangle=p_{A}(-1)^{L_{A}-\Lambda_{A}}\left|L_{A},-\Lambda_{A}\right\rangle,
$$

where $p_{A}$ is the parity of the atomic state $A$. Using the analogous relation for atom $B$ and the symmetry property of Clebsch-Gordan coefficients

$$
\left\langle j_{1} m_{1} j_{2} m_{2} \mid j m\right\rangle=(-1)^{j_{1}+j_{2}-j}\left\langle j_{1},-m_{1}, j_{2},-m_{2} \mid j,-m\right\rangle,
$$

we find

$$
\hat{\sigma}_{v}\left|\left(L_{A} L_{B}\right) L \Lambda\right\rangle=p_{A} p_{B}(-1)^{L-\Lambda}\left|\left(L_{A} L_{B}\right) L,-\Lambda\right\rangle .
$$

For $\Lambda=0$ ( $\Sigma$ states) we find that depending on the parities of the atoms there are $L_{<} \Sigma^{+}$states and $\left(L_{<}+1\right) \Sigma^{-}$states, or vice versa. Furthermore, from the invariance of the interaction operator under reflection, $\hat{\sigma}_{v} \hat{V}^{S} \hat{\sigma}_{v}=\hat{V}^{S}$, we find that the matrix elements for negative $\Lambda$ are related to the positive $\Lambda$ elements by

$$
V_{L \Lambda ; L^{\prime} \Lambda}^{S}=(-1)^{L+L^{\prime}} V_{L,-\Lambda ; L^{\prime},-\Lambda}^{S} .
$$

Hence, the $\Sigma^{ \pm}$states require $L_{<}\left(L_{<}+1\right) / 2+\left(L_{<}+1\right)\left(L_{<}\right.$ $+2) / 2=\left(L_{<}+1\right)^{2}$ parameters, the states with $0<\Lambda \leqslant N$ require $N\left(2 L_{<}+1\right)\left(L_{<}+1\right)$ parameters, and for states with $\Lambda$ $>N$ we divide the result in Eq. (54) by 2 . Together, after factorization, this gives

$$
n\left(C_{\infty, v}\right)=\left(L_{<}+1\right)\left[\frac{1}{3} L_{<}\left(4 L_{<}+5\right)+1+N\left(2 L_{<}+1\right)\right] .
$$

The number of distinct Born-Oppenheimer eigenvalues is the number of $\Sigma$ states $\left(2 L_{<}+1\right)$, plus the number of states with $0<\Lambda \leqslant N$ [i.e., $N\left(2 L_{<}+1\right)$ ], plus the number of states with $\Lambda>N\left[\Sigma_{i=1}^{2 L_{<}} i=L_{<}\left(2 L_{<}+1\right)\right],{ }^{6}$

$$
n_{\mathrm{BO}}\left(C_{\infty, v}\right)=\left(2 L_{<}+1\right)\left(L_{>}+1\right) \text {. }
$$

\section{D. $D_{\infty, h}$ symmetry}

A homonuclear diatomic molecule has $D_{\infty, h}$ symmetry. We do not consider resonant interactions, so we only discuss the $L_{A}=L_{B}$ case. Equation (58) shows that $\Sigma^{+}$and $\Sigma^{-}$states correspond to even and odd $L$, respectively. States with $(-1)^{L+S}=1$ are gerade and states with $(-1)^{L+S}=-1$ are 
ungerade ${ }^{22}$ so $V_{L \Lambda ; L^{\prime} \Lambda}^{S}$ is zero unless $L+L^{\prime}$ is even. Hence, the $\Sigma_{u / g}^{ \pm}$states still require $\left(L_{A}+1\right)^{2}$ parameters, but all states with $|\Lambda|>0$ split into two blocks. To count the parameters for states with $|\Lambda|>0$ we have to split the sum for $i$ from 1 to $2 L_{A}$ in a sum over odd and a sum over even $i$. The blocks with $2 m$ states of a given $\Lambda$ split into two blocks of dimension $m$, so they require $f(2 m)=m(m+1)$ parameters. The blocks with $2 m-1$ states of a given $\Lambda$ split into a block of dimension $m$ and a block of dimension $m-1$, so they require $f(2 m-1)=m(m+1) / 2+(m-1) m / 2=m^{2}$ parameters. Hence, the states with $|\Lambda|>0$ require

$$
\sum_{m=1}^{L_{A}}[f(2 m)+f(2 m-1)]=\frac{L_{A}}{6}\left(L_{A}+1\right)\left(4 L_{A}+5\right)
$$

parameters. When we add $\left(L_{A}+1\right)^{2}$ to this we get

$$
\left.n\left(D_{\infty, h}\right)=\left(L_{A}+1\right)\left[\frac{L_{A}}{6}\left(4 L_{A}+11\right)+1\right)\right] .
$$

\section{E. Symmetry of the $V_{k_{1} k_{2} k}^{S}(R)$ coefficients}

The symmetry properties of the space-fixed parameters $V_{k_{1} k_{2} k}^{S}$ may be derived from the properties of the body-fixed parameters $V_{L \Lambda ; L^{\prime} \Lambda}^{S}(R)$ and the transformation in Eq. (51). Alternatively, we may require the effective potential of Eqs. (48) and (49) to be a Hermitian operator, invariant under inversion $(\hat{i})$ and time reversal $(\hat{\theta})$. Space-fixed spin functions are invariant under inversion. The spin-projection part of Eq. (48) is also invariant under inversion, because it can be rewritten in space-fixed functions

$$
\sum_{\Sigma}|S \Sigma\rangle\left\langle S \Sigma\left|=\sum_{M_{S}}\right| S M_{S}\right\rangle\left\langle S M_{S}\right| .
$$

For the orbital part we have

$$
\hat{i}\left|L_{A} M_{A}\right\rangle=p_{A}\left|L_{A} M_{A}\right\rangle,
$$

where the parity $p_{A}$ was first introduced in Eq. (56) and we find that $\hat{T}_{k_{1} q_{1}}\left(L_{A}\right)$ and $\hat{T}_{k_{2} q_{2}}\left(L_{B}\right)$ are invariant under inversion too. Combining these results with

$$
\hat{i} C_{k q}^{*}(\hat{\boldsymbol{R}})=(-1)^{k} C_{k q}^{*}(\hat{\boldsymbol{R}}),
$$

we find that

$$
\hat{i} \hat{V} \hat{i}^{\dagger}=\hat{V}
$$

is satisfied when $k$ is even.

The time-reversal operator is antiunitary. ${ }^{23}$ When applied to a space-fixed spin function it gives ${ }^{25}$

$$
\hat{\theta}\left|S M_{S}\right\rangle=(-1)^{S-M_{S}}\left|S,-M_{S}\right\rangle .
$$

The spin projector is invariant under time reversal because

$$
\begin{aligned}
\hat{\theta} \sum_{M_{S}}\left|S M_{S}\right\rangle\left\langle S M_{S}\right| \hat{\theta}^{\dagger} & =\sum_{M_{S}}(-1)^{2\left(S-M_{S}\right)}\left|S,-M_{S}\right\rangle\left\langle S,-M_{S}\right| \\
& =\sum_{M_{S}}\left|S M_{S}\right\rangle\left\langle S M_{S}\right| .
\end{aligned}
$$

For the orbital part $\hat{\theta}$ corresponds to complex conjugation, and in the Condon and Shortley phase convention

$$
\hat{\theta}\left|L_{A} M_{A}\right\rangle=p_{A}(-1)^{L_{A}-M_{A}}\left|L_{A},-M_{A}\right\rangle,
$$

from which we derive

$$
\hat{\theta} \hat{T}_{k_{1} q_{1}}\left(L_{A}\right) \hat{\theta}^{\dagger}=(-1)^{k_{1}-q_{1}} \hat{T}_{k_{1},-q_{1}}\left(L_{A}\right) .
$$

This result, together with

$$
C_{k q}=(-1)^{q} C_{k,-q}^{*}
$$

and Eq. (57), shows that the condition

$$
\hat{\theta} \hat{V} \hat{\theta}^{\dagger}=\hat{V}
$$

is satisfied when

$$
\left(V_{k_{1} k_{2} k}^{S}\right)^{*}=(-1)^{k} V_{k_{1} k_{2} k}^{S}
$$

and because $k$ is even, the expansion coefficients must be real.

From Eqs. (35), (57), and (72) we may derive that the condition

$$
\hat{V}^{\dagger}=\hat{V}
$$

results in

$$
\left(V_{k_{1} k_{2} k}^{S}\right)^{*}=(-1)^{k_{1}+k_{2}} V_{k_{1} k_{2} k}^{S}
$$

and since the expansion coefficients are real $k_{1}+k_{2}$ must be even.

From the triangular conditions we have $k=\left|k_{1}-k_{2}\right|, \mid k_{1}$ $-k_{2} \mid+2, \ldots, k_{1}+k_{2}$. Notice that $C_{\infty, v}$ symmetry is implicit in Eq. (49). For a homonuclear molecule ( $D_{\infty, h}$ symmetry) we get the additional relation

$$
V_{k_{1} k_{2} k}^{S}=V_{k_{2} k_{1} k}^{S} .
$$

\section{RELATIONS BETWEEN LONG-RANGE POTENTIALS}

In the limit of large interatomic separation, the interaction potential between two non- $S$-state atoms is dominated by first order terms and the $\mathrm{ABO}$ potentials correlating with certain atomic limits are proportional to products of the permanent multipole moments of the atoms. Certain linear combinations of these $\mathrm{BO}$ potentials must vanish, which could be used to verify the results of ab initio supermolecular calculations or to fit the potentials with functional forms that asymptotically satisfy the required relations. Similarly, the second order corrections to the different $\mathrm{ABO}$ potentials may be interrelated if the orbital angular momenta of the interacting atoms are sufficiently large. In this section, we analyze the constraints on the adiabatic interaction potentials and discuss these relations.

In the long range the effective interaction potential (48)-(51) for neutral atoms can be expanded in powers of $1 / R$, 
TABLE II. The number of independent parameters required to describe the long-range interaction between two open shell atoms with the same atomic term $\left(L_{A}=L_{B}\right)$ in a homonuclear molecule $\left(D_{\infty, h}\right)$ and in a heteronuclear molecule $\left(C_{\infty, v}\right)$. The columns labeled $C_{n}$ give the number of independent parameters required to compute all $C_{n\left(k_{1}, k_{2}\right) k}$ coefficients. The columns labeled "max" give the maximum number of independent parameters for the given symmetry and $N_{\mathrm{BO}}$ is the number of adiabatic Born-Oppenheimer potentials for a given spin multiplicity.

\begin{tabular}{|c|c|c|c|c|c|c|c|c|c|c|c|}
\hline \multirow[b]{2}{*}{$L_{A}$} & \multirow[b]{2}{*}{$N_{\text {BO }}$} & \multicolumn{5}{|c|}{$D_{\infty, h}$} & \multicolumn{5}{|c|}{$C_{\infty, v}$} \\
\hline & & $C_{5}$ & $C_{6}$ & $C_{7}$ & $C_{8}$ & $\max$ & $C_{5}$ & $C_{6}$ & $C_{7}$ & $C_{8}$ & $\max$ \\
\hline 0 & 1 & 0 & 1 & 0 & 1 & 1 & 0 & 1 & 0 & 1 & 1 \\
\hline 1 & 6 & 1 & 7 & 0 & 7 & 7 & 1 & 8 & 0 & 8 & 8 \\
\hline 2 & 15 & 1 & 7 & 1 & 13 & 22 & 1 & 8 & 2 & 20 & 29 \\
\hline 3 & 28 & 1 & 7 & 1 & 13 & 50 & 1 & 8 & 2 & 20 & 72 \\
\hline 4 & 45 & 1 & 7 & 1 & 13 & 95 & 1 & 8 & 2 & 20 & 145 \\
\hline
\end{tabular}

$$
\hat{V}=\sum_{n=5}^{\infty} \frac{\hat{V}^{(n)}}{R^{n}}
$$

where each of the terms $V^{(n)}$ can be represented by the tensorial expansion

$$
V^{(n)}=\sum_{k_{1} k_{2} k} V_{k_{1} k_{2} k}^{(n)} \sum_{q}\left[\hat{T}_{k_{1}}\left(L_{\mathrm{A}}\right) \otimes \hat{T}_{k_{2}}\left(L_{\mathrm{B}}\right)\right]_{q}^{(k)} C_{k q}^{*}(\hat{\boldsymbol{R}}) .
$$

Odd- $n$ terms are given in first order and even- $n$ terms in second order perturbation theory. ${ }^{26}$ Third order contributions start at $n=9$. For neutral atoms the summation begins at $n$ $=5$. We will use the explicit expressions for first and second order contributions derived in Secs. II and III to determine the number of independent parameters for $n=5,6,7$, or 8 . When these numbers are less than the maximum number of independent parameters derived for the given symmetry we obtain one or more linear restrictions on the corresponding body-fixed potentials $V_{L \Lambda ; L^{\prime} \Lambda}^{(n)}(R)$ through Eq. (51), from which we derive restrictions on $\mathrm{ABO}$ potentials.

\section{A. Coefficients $c_{n,\left(k_{1} k_{2}\right) k}^{(1)}$ with odd $n$}

The expressions for the first order interaction are given in Eq. (16) and the expansion coefficients $c_{n,\left(k_{1} k_{2}\right) k}^{(1)}$ are given in Eq. (17) (with $k_{1}=l_{1}, k_{2}=l_{2}$, and $k=L_{12}$ ). Recall that $L_{12}$ is restricted to $l_{1}+l_{2}$ and that for atoms both $l_{1}$ and $l_{2}$ must be even because of parity conservation and in addition $l_{1}$ $\leqslant 2 L_{A}$ and $l_{2} \leqslant 2 L_{B}$ and $n=l_{1}+l_{2}+1$. Hence, for neutral atoms the leading term with $n=5$ is determined by a single parameter,

$$
c_{5,(2,2) 4}^{(1)}=\sqrt{70} Q_{2}^{(A)}\left(L_{A}\right) Q_{2}^{(B)}\left(L_{B}\right) .
$$

For $n=7$ there are two parameters, $c_{7,(2,4) 6}^{(1)}$ and $c_{7,(4,2) 6}^{(1)}$, which are identical in the case of $D_{\infty, h}$ symmetry.

In Tables II $\left(L_{A}=L_{B}\right)$ and III $\left(L_{A}<L_{B}\right)$ we list the number of parameters for each $n$ for several values of $L_{A}$ and $L_{B}$, together with the maximum number of parameters for the given symmetry.

\section{B. Coefficients $c_{n,\left(k_{1} k_{2}\right) k}^{(2)}$ with even $n$}

The expression for the second order interaction is given in Eq. (37) and the dispersion and induction coefficients are given in Eqs. (39) and (46). The value of $n$ is given by $l_{1}$ $+l_{2}+l_{1}^{\prime}+l_{2}^{\prime}+2$. For the induction coefficient $c^{\text {(ind,A) }}, l_{1}+l_{1}^{\prime}, l_{2}$, and $l_{2}^{\prime}$ have to be even as the polarizability operator for atom $A$ must have even parity and the odd rank permanent multipole moments vanish. For the dispersion interaction only the sums $l_{1}+l_{1}^{\prime}$ and $l_{2}+l_{2}^{\prime}$ must be even. The general restrictions on $k_{1}, k_{2}$, and $k$ were given in Sec. IV E. From the explicit expressions we find the additional constraints $k_{1} \leqslant \min \left(l_{1}\right.$ $\left.+l_{1}^{\prime}, 2 L_{A}\right), k_{2} \leqslant \min \left(l_{2}+l_{2}^{\prime}, 2 L_{B}\right)$. The number of independent parameters is given in Tables II and III.

\section{EXAMPLES}

\section{A. Interaction of an open shell atom with $L_{A}>0$ and an S-state atom}

When one of the atoms is in a state with $L_{B}=0$, the molecular states defined in Eq. (50) are uniquely labeled by $\Lambda$, since $L=L_{A}$. With the notation $V_{\Lambda} \equiv V_{L \Lambda ; L \Lambda}$ and $L_{B}=0$ Eq. (51) simplifies to (see Appendix B)

$$
V_{k 0 k}=\frac{[k]^{1 / 2}}{[L]^{1 / 2}} \sum_{\Lambda=-L}^{L}\langle L \Lambda k 0 \mid L \Lambda\rangle V_{\Lambda}
$$

and Eq. (49) simplifies to (see Appendix B)

$$
\hat{V}=\sum_{k} V_{k 0 k} \sum_{q} \hat{T}_{k}\left(L_{A}\right) C_{k q}^{*}(\hat{\boldsymbol{R}}) .
$$

Since $k$ is even (see Sec. IV E) we may replace the spherical tensor by an effective one-electron potential proportional to a

TABLE III. Same as in Table II for the case with $L_{A}<L_{B}$ and $C_{\infty, v}$ symmetry.

\begin{tabular}{rrrrrrrr}
\hline \hline$L_{A}$ & $L_{B}$ & $N_{\text {BO }}$ & $C_{5}$ & $C_{6}$ & $C_{7}$ & $C_{8}$ & $\max$ \\
\hline 0 & 1 & 2 & 0 & 2 & 0 & 2 & 2 \\
0 & 2 & 3 & 0 & 2 & 0 & 3 & 3 \\
0 & 3 & 4 & 0 & 2 & 0 & 3 & 4 \\
0 & 4 & 5 & 0 & 2 & 0 & 3 & 5 \\
1 & 2 & 9 & 1 & 8 & 1 & 14 & 14 \\
1 & 3 & 12 & 1 & 8 & 1 & 14 & 20 \\
1 & 4 & 15 & 1 & 8 & 1 & 14 & 26 \\
2 & 3 & 20 & 1 & 8 & 2 & 20 & 44 \\
2 & 4 & 25 & 1 & 8 & 2 & 20 & 59 \\
3 & 4 & 35 & 1 & 8 & 2 & 20 & 100 \\
\hline \hline
\end{tabular}


spherical harmonic $Y_{k q}\left(\hat{\mathbf{r}}_{A}\right)$ [see Eq. (21) in Ref. 14] and the states $\left|L_{A} M_{A}\right\rangle$ by spherical harmonics $Y_{L_{A} M_{A}}\left(\hat{\mathbf{r}}_{A}\right)$. Using the spherical harmonic addition theorem (see Appendix B) we obtain the Legendre expansion

$$
V\left(\hat{\boldsymbol{r}}_{A}, \hat{\boldsymbol{R}}\right)=\sum_{k} V_{k} P_{k}\left(\hat{\boldsymbol{r}}_{A} \cdot \hat{\boldsymbol{R}}\right),
$$

with

$$
V_{k}=V_{k 0 k} \frac{[k]^{1 / 2}}{[L]^{1 / 2}}\langle L 0 k 0 \mid L 0\rangle^{-1} .
$$

By substituting Eq. (81) into this equation we relate $V_{k}$ to the molecular potentials

$$
V_{k}=\frac{[k]}{[L]} \sum_{\Lambda=-L}^{L} \frac{\langle L \Lambda k \Lambda \mid L \Lambda\rangle}{\langle L 0 k 0 \mid L 0\rangle} V_{\Lambda}
$$

This result is identical to Eq. (3) of Ref. 12 or Eq. (2.12) of Ref. 13. This expression shows that the $L+1$ adiabatic molecular potentials $\left\{V_{\Lambda}, \Lambda=0,1, \ldots, L\right\}$ are related to $L+1$ expansion coefficients $\left\{V_{k}, k=0,2, \ldots, 2 L\right\}$.

With one atom in an $S$ state there is no electrostatic long-range interaction in first order and only potential terms with even $k$ appear in the potential. From the explicit expressions of the dispersion $\left(c_{n,(k 0) k}^{\text {(disp) }}\right)$ and induction $\left(c_{n, k 0) k}^{\text {(ind,B) }}\right)$ coefficients we find that only coefficients with $k \leqslant \min (n$ $-4,2 L)$ are nonzero [use $n=l_{1}+l_{1}^{\prime}+l_{2}+l_{2}^{\prime}+2, k \leqslant \min \left(l_{1}\right.$ $\left.+l_{1}^{\prime}, 2 L\right)$ in $\alpha_{\left(l_{1}, l_{1}^{\prime}\right) k_{1}}^{(A)}$, and assume $l_{2}+l_{2}^{\prime}$ is at least 2 for neutral atoms]. Hence, whenever $2 L>n-4$ there are $L-(n-4) / 2$ linear constraints on the second order interaction coefficients. For example, for a $D$ state Eq. (85) gives

$$
\begin{aligned}
& V_{0}=\frac{1}{5}\left(V_{\Sigma}+2 V_{\Pi}+2 V_{\Delta}\right), \\
& V_{2}=\left(V_{\Sigma}-V_{\Delta}\right)+\left(V_{\Pi}-V_{\Delta}\right), \\
& V_{4}=\frac{3}{5}\left[3\left(V_{\Sigma}-V_{\Pi}\right)+\left(V_{\Delta}-V_{\Pi}\right)\right] .
\end{aligned}
$$

and for $F$ states the relations are

$$
\begin{aligned}
& V_{0}=\frac{1}{7}\left(V_{\Sigma}+2 V_{\Pi}+2 V_{\Delta}+2 V_{\Phi}\right), \\
& V_{2}=\frac{5}{14}\left[2\left(V_{\Sigma}-V_{\Phi}\right)+3\left(V_{\Pi}-V_{\Phi}\right)\right], \\
& V_{4}=\frac{3}{7}\left[3\left(V_{\Sigma}-V_{\Delta}\right)+\left(V_{\Pi}-V_{\Delta}\right)+3\left(V_{\Phi}-V_{\Delta}\right)\right], \\
& V_{6}=\frac{13}{70}\left[10\left(V_{\Sigma}-V_{\Pi}\right)+5\left(V_{\Delta}-V_{\Pi}\right)+\left(V_{\Delta}-V_{\Phi}\right)\right] .
\end{aligned}
$$

The substitution of the $C_{6}$ coefficients of the adiabatic molecular potentials in these expressions must yield $V_{k>2}=0$. Similarly, $V_{k>4}=0$ for the $C_{8}$ coefficients. Thus, we find that at interatomic distances where terms with $n>6$ are negligible there is one linear constraint on the $\mathrm{ABO}$ potentials for the interaction of an $S$-state atom with a $D$-state atom, and there are two linear constraints for an $S$-state atom interacting with an $F$-state atom, etc.

\section{B. The interaction of two P-state atoms}

The row with $L_{A}=1$ in Table II shows that there are six ABO potentials correlating with the atomic states $\left(N_{\mathrm{BO}}=6\right)$. These states are listed explicitly in the first three rows in Table I $(L=0,1,2)$. In a homonuclear molecule there are only two states of the same symmetry (the $\Sigma^{+}$states), so the number of potentials $V_{L \Lambda ; L^{\prime} \Lambda}(R)$ is equal to $N_{\mathrm{BO}}+1=7$. In the heteronuclear case the $g / u$ label is dropped so there is an additional off-diagonal element between the two $\Pi$ states $\left[V_{1,1 ; 2,1}(R)\right]$.

With the compact notation $V_{\Lambda}\left(L, L^{\prime}\right) \equiv V_{L \Lambda ; L^{\prime} \Lambda}$ and $V_{\Lambda}(L) \equiv V_{\Lambda}(L, L)$ the eight space-fixed expansion coefficients $V_{k_{1} k_{2} k}$ are given by

$$
\begin{aligned}
V_{000}= & \frac{1}{3}\left[V_{\Sigma}(0)+V_{\Sigma}(1)+V_{\Sigma}(2)+2 V_{\Pi}(1)+2 V_{\Pi}(2)\right. \\
& \left.+2 V_{\Delta}(2)\right],
\end{aligned}
$$

$$
\begin{aligned}
V_{110}= & \frac{1}{2 \sqrt{3}}\left[2 V_{\Sigma}(0)+V_{\Sigma}(1)-V_{\Sigma}(2)+2 V_{\Pi}(1)-2 V_{\Pi}(2)\right. \\
& \left.-2 V_{\Delta}(2)\right] \\
V_{220}= & \frac{1}{6 \sqrt{5}}\left[10 V_{\Sigma}(0)-5 V_{\Sigma}(1)+V_{\Sigma}(2)-10 V_{\Pi}(1)\right. \\
& \left.+2 V_{\Pi}(2)+2 V_{\Delta}(2)\right]
\end{aligned}
$$$$
V_{022}=\frac{1}{3 \sqrt{2}}\left[2 \sqrt{2} V_{\Sigma}(0,2)+V_{\Sigma}(1)-V_{\Sigma}(2)-V_{\Pi}(1)\right.
$$$$
\left.-6 V_{\Pi}(1,2)-V_{\Pi}(2)+2 V_{\Delta}(2)\right] \text {, }
$$

$$
V_{112}=\frac{1}{\sqrt{6}}\left[-\sqrt{2} V_{\Sigma}(0,2)-V_{\Sigma}(1)-V_{\Sigma}(2)+V_{\Pi}(1)\right.
$$$$
\left.-V_{\Pi}(2)+2 V_{\Delta}(2)\right]
$$$$
V_{202}=\frac{1}{3 \sqrt{2}}\left[2 \sqrt{2} V_{\Sigma}(0,2)+V_{\Sigma}(1)-V_{\Sigma}(2)-V_{\Pi}(1)\right.
$$$$
\left.+6 V_{\Pi}(1,2)-V_{\Pi}(2)+2 V_{\Delta}(2)\right] \text {, }
$$

$$
\begin{aligned}
V_{222}= & \frac{1}{3 \sqrt{14}}\left[7 \sqrt{2} V_{\Sigma}(0,2)-7 V_{\Sigma}(1)+V_{\Sigma}(2)+7 V_{\Pi}(1)\right. \\
& \left.+V_{\Pi}(2)-2 V_{\Delta}(2)\right],
\end{aligned}
$$

$$
V_{224}=\sqrt{\frac{2}{35}}\left[3 V_{\Sigma}(2)-4 V_{\Pi}(2)+V_{\Delta}(2)\right] .
$$

For homonuclear molecules only terms with even combinations $L+L^{\prime}$ are allowed so $V_{\Pi}(1,2)=0$ and $V_{202}=V_{022}$. The leading term in the first order long-range interaction, $V_{L \Lambda ; L^{\prime} \Lambda}^{(5)} / R^{5}$, is proportional to $\left\langle L, \Lambda, L^{\prime},-\Lambda \mid 4,0\right\rangle$ [See Eq. (8) in Ref. 27]. Hence, the only nonzero contributions in this term arise from the diagonal potentials with $L=L^{\prime}=2$. The $C_{5}$ coefficients for the interaction potentials between two $\mathrm{O}\left({ }^{3} P\right)$ atoms were given in Ref. 22. Substitution of the $C_{5}$ 
coefficients in Eq. (88) confirms that all terms $V_{k_{1} k_{2} k}$ except $V_{224}$ vanish.

In Table II we find that already the leading term in second order $\left(C_{6}\right)$ makes all 7 (in $D_{\infty, h}$ symmetry) or 8 (in $C_{\infty, v}$ symmetry) potentials independent. The two $\Sigma$ states that asymptotically correlate with $L=0$ and $L=2$ are eigenfunctions of the 2 by 2 matrix

$$
\begin{aligned}
\left(\begin{array}{cc}
V_{\Sigma}(0) & V_{\Sigma}(0,2) \\
V_{\Sigma}(2,0) & V_{\Sigma}(2)
\end{array}\right)= & R^{-5}\left(\begin{array}{cc}
0 & 0 \\
0 & V_{\Sigma}^{(5)}(2)
\end{array}\right) \\
& +R^{-6}\left(\begin{array}{cc}
V_{\Sigma}^{(6)}(0) & V_{\Sigma}^{(6)}(0,2) \\
V_{\Sigma}^{(6)}(2,0) & V_{\Sigma}^{(6)}(2)
\end{array}\right) \\
& +\cdots .
\end{aligned}
$$

By solving for the eigenvalues of this matrix using perturbation theory, with the $R^{-6}$ term as the perturbation we observe that the $V_{\Sigma}^{(6)}(0,2)$ coupling contributes to terms proportional to $R^{-7}$ and higher powers of $1 / R$ in the ABO potentials. To calculate the $V_{\Sigma}(0,2)$ potential, one needs information about the $\mathrm{ABO}$ eigenfunctions, e.g., by integrating the radial derivative coupling from infinity inwards (see, e.g., Ref. 28).

Other examples will be discussed in a separate paper, where we present numerical results for long-range interactions involving the $\operatorname{Sc}\left({ }^{1} D\right)$ and $\operatorname{Ti}\left({ }^{3} F\right)$ atoms. ${ }^{17}$

\section{CONCLUSION}

Reference 14 presents a general tensorial expansion for the anisotropic electronic interaction between two open shell atoms. In the present paper we have derived explicit expression for the expansion coefficients in the long range in first and second order perturbation theory. We have demonstrated that in first order, and also in second order when one of the atoms is in an $S$ state, the interaction can be represented by a spherical harmonic expansion analogous to the expansion of the potential for two homonuclear diatomic molecules. The second order interaction between two atoms with nonzero orbital angular momenta, however, requires odd rank spherical tensors that are invariant under space inversion.

We have analyzed the transformation of the interaction potential between two non- $S$-state atoms from the body-fixed to the space-fixed frame [Eq. (51)] and the relation between the body-fixed expansion coefficients and the molecular adiabatic potentials. When one of the atoms is in an $S$ state, the body-fixed expansion coefficients are identical to the adiabatic potentials. This is also true in some other special cases, e.g., in the first order interaction between two $P$-state atoms. In general, however, when more than one $\mathrm{ABO}$ potential of the same symmetry exist, the transformation involves a diagonalization.

By using the multipole expansion of the Coulomb operator in the long range we have obtained a $R^{-n}$ expansion of the potential expansion coefficients. When the $R^{-n}$ expansion is truncated some terms in the spherical tensor expansion of the interaction potential become zero. The truncation results in linear or, when a diagonalization is involved, nonlinear constraints on the adiabatic Born-Oppenheimer potentials. We have presented a detailed analysis of the number of independent parameters in the long-range potentials, both for homonuclear and heteronuclear molecules.

Our results can be used to verify quantum chemistry calculations of the interaction potentials between atoms with nonzero values of the electronic orbital angular momenta at large interatomic separations. This work also provides the functional forms that can be used to fit interatomic interaction potentials at long range. Our derivation of explicit expressions for the dispersion and induction interaction coefficients in terms of (dynamic) polarizabilities demonstrates that a complete description of interatomic interactions must include both the observable scalar and even rank tensor atomic polarizabilities and the "out-of-phase" odd rank polarizability operators defined in Eq. (26).

The long-range coefficients defined in this paper computed at the time-dependent density functional theory level for $\mathrm{Sc}-\mathrm{Sc}, \mathrm{Sc}-\mathrm{Ti}$, and Ti-Ti will be presented in a separate paper. $^{17}$

\section{ACKNOWLEDGMENTS}

The authors thank Professor Ad van der Avoird and Professor Alex Dalgarno for carefully reading the manuscript. The work of one of the authors (R.V.K.) was supported by the Natural Sciences and Engineering Research Council (NSERC) of Canada. Another author (X.C.) acknowledges the support of the Chemical Sciences, Geosciences and Biosciences Division of the Office of Basic Energy Sciences, Office of Science, U.S. Department of Energy, the National Science Foundation Grant No. EPS-0346458, and the Office of the Vice President for Research and Development of the University of Montana.

\section{APPENDIX A: SUM FORMULAS}

To derive Eq. (54) we use the sum formulas

$$
\begin{aligned}
& \sum_{i=0}^{n} i=n(n+1) / 2, \\
& \sum_{i=0}^{n} i^{2}=n(n+1)(2 n+1) / 6,
\end{aligned}
$$

from which we obtain

$$
\sum_{i=0}^{n} i(i+1)=n(n+1)(n+2) / 3 .
$$

\section{APPENDIX B: INTERACTION BETWEEN AN S-STATE ATOM AND A NON-S-STATE ATOM}

When $L_{\mathrm{B}}=0$ in Eq. (49), $k_{2}$ and $q_{2}$ must be zero, $\hat{T}_{k_{2} q_{2}}\left(L_{B}=0\right)=|00\rangle\langle 00|=1$, and we obtain Eq. (82). Equation (81) follows from Eq. (51) given that 


$$
\left\{\begin{array}{lll}
L & L & k \\
0 & 0 & 0 \\
L & L & k
\end{array}\right\}=[L]^{-1}[k]^{-1 / 2} .
$$

To derive the Legendre expansion in Eq. (83) from (82) for $k$ even we rewrite Eqs. (21) and (23) from Ref. 14 as

$$
T_{k q}(L)=C_{k q}\left(\hat{\boldsymbol{r}}_{A}\right) \frac{[k]^{1 / 2}}{[L]^{1 / 2}}\langle L 0 k 0 \mid L 0\rangle^{-1} .
$$

Substituting this expression into Eq. (82) and using the spherical harmonic addition theorem

$$
P_{k}\left(\hat{\boldsymbol{R}} \cdot \hat{\boldsymbol{r}}_{\mathrm{A}}\right)=\sum_{q} C_{k q}^{*}(\hat{\boldsymbol{R}}) C_{k q}\left(\hat{\boldsymbol{r}}_{\mathrm{A}}\right),
$$

we obtain Eq. (83).

${ }^{1}$ C. I. Hancox, S. C. Doret, M. T. Hummon, R. V. Krems, and J. M. Doyle, Phys. Rev. Lett. 94, 013201 (2005).

${ }^{2}$ R. V. Krems, J. Kłos, M. F. Rode, M. M. Szczȩśniak, G. Chałasiński, and A. Dalgarno, Phys. Rev. Lett. 94, 013202 (2005).

${ }^{3}$ C. I. Hancox, S. C. Doret, M. T. Hummon, L. Luo, and J. M. Doyle, Nature (London) 431, 281 (2004).

${ }^{4}$ R. V. Krems, Int. Rev. Phys. Chem. 24, 99 (2005).

${ }^{5}$ M. Marinescu and A. Dalgarno, Phys. Rev. A 52, 311 (1995).

${ }^{6}$ E. Wigner and E. E. Witmer, Zeitschrift für Physik 51, 859 (1928).

${ }^{7}$ J. Callaway and E. Bauer, Phys. Rev. 140, A1072 (1965).

${ }^{8}$ F. Rebentrost and W. A. Lester, Jr., J. Chem. Phys. 64, 3879 (1976).
${ }^{9}$ M.-L. Dubernet and J. M. Hutson, J. Chem. Phys. 101, 1939 (1994).

${ }^{10}$ W. B. Zeimen, J. A. Kłos, G. C. Groenenboom, and A. van der Avoird, J. Chem. Phys. 118, 7340 (2003).

${ }^{11}$ T. A. Grinev, T. V. Tscherbul, A. A. Buchachenko, S. Cavalli, and V. Aquilanti, J. Phys. Chem. A 110, 5458 (2006).

${ }^{12}$ X. Chu, A. Dalgarno, and G. C. Groenenboom, Phys. Rev. A 72, 032703 (2005).

${ }^{13}$ V. Aquilanti and G. Grossi, J. Chem. Phys. 73, 1165 (1980).

${ }^{14}$ R. V. Krems, G. C. Groenenboom, and A. Dalgarno, J. Phys. Chem. A 108, 8941 (2004).

${ }^{15}$ R. Santra and C. H. Greene, Phys. Rev. A 67, 062713 (2003).

${ }^{16}$ A. J. Stone, The Theory of Intermolecular Forces (Oxford University Press, Oxford, 1996).

${ }^{17}$ X. Chu and G. C. Groenenboom (unpublished).

${ }^{18}$ P. E. S. Wormer, Ph.D. thesis, University of Nijmegen, 1975.

${ }^{19}$ R. P. Leavitt, J. Chem. Phys. 72, 3472 (1980).

${ }^{20} \mathrm{~K}$. Blum, Density Matrix Theory and Applications, Physics of Atoms and Molecules (Plenum, New York, 1981).

${ }^{21}$ H. B. G. Casimir and D. Polder, Phys. Rev. 73, 360 (1948).

${ }^{22}$ B. Zygelman, A. Dalgarno, and R. D. Sharma, Phys. Rev. A 50, 3920 (1994).

${ }^{23}$ A. Messiah, Quantum Mechanics (North Holland, Amsterdam, 1969).

${ }^{24}$ M. C. G. N. van Vroonhoven and G. C. Groenenboom, J. Chem. Phys. 117, 5240 (2002)

${ }^{25}$ D. M. Brink and G. R. Satchler, Angular Momentum, 3rd ed. (Clarendon, Oxford, 1993).

${ }^{26}$ T. Y. Chang, Rev. Mod. Phys. 39, 911 (1967).

${ }^{27}$ M. C. G. N. van Vroonhoven and G. C. Groenenboom, J. Chem. Phys. 116, 1954 (2002).

${ }^{28}$ M. C. G. N. van Vroonhoven and G. C. Groenenboom, J. Chem. Phys. 116, 1965 (2002). 